\title{
Consorciação de taro e crotalária manejada com corte rente ao solo e poda na altura do dossel
}

\author{
Mário Puiatti*l, Nelson Licínio Campos de Oliveira ${ }^{2}$, Paulo Roberto Cecon ${ }^{3}$, Aline da Silva Bhering ${ }^{4}$ \\ http://dx.doi.org/10.1590/0034-737X201562030007
}

\section{RESUMO}

A associação de culturas com leguminosas de adubação verde visa à manutenção ou melhorias da fertilidade do solo. Com o objetivo de avaliar a consorciação do taro 'Japonês' com crotalária, foram conduzidos dois experimentos, em Viçosa, MG, no período de 10/09/2010 a 10/06/2011. No Experimento I, as plantas de crotalária foram cortadas rente ao solo e, no Experimento II, podadas à altura do dossel do taro; em ambos, os tratamentos consistiram em seis épocas de corte ou poda da crotalária $(75 ; 105 ; 135 ; 165 ; 195$ e 225 dias após a semeadura da crotalária - DAS), mais a monocultura do taro. As partes cortadas ou podadas foram depositadas sobre o solo e determinaramse as quantidades de fitomassa e de nutrientes da crotalária. Na colheita do taro, avaliaram-se a produção de classes de rizomas e as alterações químicas do solo. Os cortes realizados após 105 DAS proporcionaram menor produção em massa de rizomas-mães e, em número, por planta, de rizomas-filhos grandes e comerciais, em comparação com os do controle. Comportamento semelhante foi observado para experimento com poda à altura do dossel. Os cortes ou podas realizados aos 135 e 165 DAS foram os que apresentaram maiores quantidades de fitomassas fresca e seca de crotalária, de carbono orgânico e de nutrientes. Aos 165 DAS, o aporte de N ao solo pela crotalária cortada chegou a $308 \mathrm{~kg} \mathrm{ha}^{-1} \mathrm{e}$, pela crotalária podada, a $202 \mathrm{~kg} \mathrm{ha}^{-1}$. A associação entre crotalária e taro é viável, sendo indicada a manutenção da consorciação até aos 105 DAS da crotalária.

Palavras-chave: Colocasia esculenta, Crotalaria juncea, consórcio, adubação verde, cobertura de solo.

\begin{abstract}
Intercropping of taro and sunnhemp managed by cutting to the ground and pruning at canopy

The association of crops with green manure legumes is a technique to promote improvement or maintenance of soil fertility. The intercrop of taro 'Japonês' with sunnhemp were evaluated in two experiments: Experiment I, sunnhemp plants were cut at ground level; and Experiment II, sunnhemp plants were pruned at the canopy height of taro. Both esperiments evaluated the effect of six times of cutting / pruning sunnhemp $(75 ; 105 ; 135 ; 165 ; 195$ and 225 days after sowing - DAS), as well as a single crop of taro (without sunnhemp). Plant clippings were placed on the ground and q mass and nutrients provided by sunnhemp were determined. At taro harvest, the production of classes of rhizomes and chemical changes in the soil were evaluated. The cuttings after 105 DAS provided less mass production of mother and daughter rhizomes and number/plant of large and commercial cormels compared with the control. Pruning at canopy height at 135 and 165 DAS provided the largest amounts of fresh and dry mass
\end{abstract}

\footnotetext{
Submetido em 24/03/2014 e aprovado em 28/05/2015

${ }^{1}$ Universidade Federal de Viçosa, Departamento de Fitotecnia, Viçosa, Minas Gerais, Brasil. mpuiatti@ufv.br

${ }^{2}$ Instituto Federal Norte de Minas Gerais, Montes Claros, Minas Gerais, Brasil. nelson.oliveira@ifnmg.edu.br

${ }^{3}$ Universidade Federal de Viçosa, Departamento de Estatística, Viçosa, Minas Gerais, Brasil. cecon@ufv.br

${ }^{4}$ Universidade Federal de Viçosa, Departamento de Fitotecnia, Viçosa, Minas Gerais, Brasil. alinebhering @ hotmail.com

*Autor para correspondência: mpuiatti@ufv.br
} 
of sunnhemp, organic carbon and nutrients. At 165 DAS, the contribution of $\mathrm{N}$ to the soil by sunnhemp cutting reached $308 \mathrm{~kg} \mathrm{ha}^{-1}$ and by sunnhemp pruning was $202 \mathrm{~kg} \mathrm{ha}^{-1}$. The intercrop of sunnhemp with taro is viable, with management of the intercropping indicated up to 105 DAS of sunnhemp.

Key words: Colocasia esculenta; Crotalaria juncea; intercropping; green manure; mulching.

\section{INTRODUÇÃO}

No Brasil, a agricultura familiar representa cerca de $85 \%$ do total de estabelecimentos agrícolas, ocupando $30 \%$ da área total. Apesar de receber apenas $25 \%$ do financiamento destinado à agricultura, a agricultura familiar tem sido responsável por cerca de $60 \%$ dos alimentos consumidos pela população brasileira e de $38 \%$ do valor bruto da produção da agropecuária nacional, além de ser a principal fonte geradora de empregos no meio rural (EMBRAPA, 2010).

Esses números mostram a importância desse segmento para a sociedade e para a economia brasileira. Todavia, são necessárias pesquisas sobre tecnologias que possam manter ou até melhorar a fertilidade do solo, a produtividade e a renda das propriedades familiares e, consequentemente, a qualidade de vida desses produtores e de seus familiares.

A agricultura familiar é constituída por pequenos e médios produtores, que diversificam as espécies cultivadas como forma de diluir custos, de aumentar a renda por unidade de área e de aproveitar a disponibilidade de mão de obra (Vandermeer, 1989). Dentre os cultivos da agricultura familiar, na região centro-sul do Brasil, está o do taro [Colocasia esculenta (L.) Schott]. O taro, denominado no centro-sul do Brasil de inhame (Puiatti \& Pereira, 2007), desempenha importante função social no Brasil, pois, além de gerar renda, é um alimento com excelentes características nutricionais, com possibilidades de uso sob diferentes formas de preparo, podendo, ainda, ser utilizado na alimentação animal, especialmente para frangos de corte (Heredia Zarate et al., 2005).

No mundo, dentre as plantas tropicais produtoras de raízes, tubérculos e rizomas, o taro figura em quarto lugar em volume produzido, vindo após a mandioca, a batata-doce e o inhame, tendo, no ano de 2012, área plantada de 1.316.985 ha, com produção de 9.967.198 Mg e produtividade de 7,57 $\mathrm{Mg} \mathrm{ha}^{-1}$ (FAO, 2013). No Brasil, Minas Gerais é o estado em que a cultura apresenta maior produtividade, alcançando média ao redor de $19 \mathrm{Mg}$ ha $^{-1}$; todavia o potencial produtivo da cultura é ainda maior, havendo a necessidade de se desenvolver novas tecnologias de cultivo.
O taro é uma espécie tropical, com ótimo de crescimento na faixa de temperatura de 21 a $27^{\circ} \mathrm{C}$ (Puiatti \& Pereira, 2007). Contudo, sob condições de alta irradiância, pode ter grande parte do limbo foliar danificado (Oliveira et al., 2004; 2006). Por ser uma planta que tolera sombreamento parcial (Gondim et al., 2007), sua consorciação com espécies de maior porte, e que propiciem sombreamento parcial, tem demonstrado ser benéfica à cultura, como é o caso do consórcio com milho verde (Puiatti et al., 2000) e do cultivo, em faixas, com crotalária - Crotalaria juncea (Oliveira et al., 2004) e guandu - Cajanus cajan (Oliveira et al., 2006).

A consorciação de culturas é um sistema muito utilizado, sobretudo nas regiões tropicais, principalmente por pequenos produtores (Vandermeer, 1989). Todavia, só recentemente os pesquisadores têm dedicado a devida atenção a essas associações, incluindo a com leguminosas. Dentre as leguminosas, a Crotalaria juncea, por ser uma planta de crescimento rápido e vigorosa, tem sido muito usada como adubo verde, visando à manutenção e, ou melhoria da fertilidade do solo.

Nos consórcios de plantas, as leguminosas têm sido das espécies mais utilizadas, principalmente como adubação verde. A adubação verde com leguminosas, além da proteção do solo contra danos ocasionados pela água de chuva e, ou, de irrigação, contribui para a nutrição das plantas pela ciclagem de nutrientes e pelo aporte de $\mathrm{N}$, por via da fixação biológica de $\mathrm{N}_{2}(\mathrm{FBN})$. Ainda, por apresentar baixa relação $\mathrm{C} / \mathrm{N}$, favorece a decomposição e mineralização por micro-organismos, contribuindo com o N para as culturas (Perin et al., 2004).

Todavia, além da espécie, a quantidade de massa e de nutrientes aportados pelo adubo verde depende das épocas de plantio e de corte ou poda e do manejo dado à massa vegetal (Souza \& Guimarães, 2013). Dessa forma, a escolha criteriosa das culturas componentes do consórcio, a época de suas respectivas instalações e de manejo da massa cortada ou podada são de fundamental importância para que se possa propiciar exploração máxima das vantagens do sistema de cultivo consorciado. Nesse aspecto, o manejo da fitomassa da associação com plantas leguminosas é importante, pois o $\mathrm{N}$ residual, provenien- 
te dos restos culturais, deve ser considerado e pode ser aproveitado pelas culturas subsequentes (Vargas, 2009; Diniz, 2011; Souza \& Guimarães, 2013).

Em razão do exposto, objetivou-se, neste trabalho, avaliar o consórcio do taro com a crotalária manejada com o corte rente ao solo e podada na altura do dossel do taro, durante o ciclo de cultivo.

\section{MATERIAL E MÉTODOS}

Foram conduzidos dois experimentos com o cultivo do taro [(Colocasia esculenta (L.) Schott)] em associação aditiva com crotalária (Crotalaria juncea L.), na Horta de Pesquisas do Departamento de Fitotecnia da Universidade Federal de Viçosa (UFV), em Viçosa, MG, no período de 10/09/2010 a 10/06/2011. O solo foi classificado como Argissolo Vermelho-Amarelo, textura argilosa, cujas características químicas são apresentadas na Tabela 1.

Cada experimento foi constituído de sete tratamentos $(\mathrm{T})$, correspondentes às épocas de corte das plantas de crotalária rente ao solo (Experimento I), ou da poda das plantas de crotalária na altura do dossel das plantas de taro (Experimento II), assim sendo: T 1 (cultivo solteiro do taro - sem crotalária), T 2 (corte ou poda da crotalária, aos 75 DAS), T 3 (corte ou poda da crotalária, aos 105 DAS), T 4 (corte ou poda da crotalária, aos 135 DAS), T 5 (corte ou poda da crotalária, aos 165 DAS), T 6 (corte ou poda da crotalária, aos 195 DAS) e T7 (corte ou poda da crotalária, aos 225 DAS).

Utilizou-se o delineamento experimental de blocos ao acaso, com quatro repetições. O preparo do solo consistiu em aração e gradagem, seguidas da abertura de sulcos com 0,12 m de profundidade, espaçados de 1,0 m. As mudas de taro foram distribuídas dentro dos sulcos a cada 0,30 m, seguindo-se do fechamento com solo. Foram utilizados como mudas, rizomas-filhos de taro 'Japonês' (BGH 5925), com massa média de 90 g, obtidos do Banco de Germoplasma de Hortaliças da UFV (BGH/UFV).

Aos 45 dias após o plantio das mudas de taro, com as plantas de taro com aproximadamente $25 \mathrm{~cm}$ de altura, procedeu-se à semeadura da crotalária. Foram distribuídas 40 sementes por metro linear, em sulcos abertos com sacho, lateralmente às fileiras das plantas de taro, à profundidade de 1,0 cm. Após 20 dias da semeadura, com as plantas de crotalária com $15 \mathrm{~cm}$ de altura, procedeu-se ao desbaste das plantas excedentes, deixando-se 20 plantas por metro linear. Dessa forma, as populações de taro e de crotalária foram, respectivamente, de 33.333 e de 200.000 plantas por hectare.

A parcela experimental consistiu em quatro fileiras espaçadas de $1,0 \mathrm{~m}$, com 3,0 $\mathrm{m}$ de comprimento, totalizando 40 plantas de taro e 240 plantas de crotalária. Considerou-se como área útil para o taro aquela ocupada pelas oito plantas centrais das duas fileiras centrais. Para avaliação da massa de crotalária, consideraram-se como úteis as plantas contidas nos $2 \mathrm{~m}$ centrais das duas fileiras centrais.

No Experimento I, em cada época de corte (tratamentos), as plantas de crotalária foram cortadas rente ao solo, na região do coleto, de forma a não permitir a sua rebrota. No Experimento II, a parte aérea das plantas de crotalária, em cada época, foram podadas na altura do dossel das plantas de taro. O material cortado ou podado, após pesagem para obtenção da fitomassa fresca, foi disposto sobre o solo, lateralmente às fileiras do taro. Nessa época, foi retirada uma amostra representativa das plantas de crotalária cortadas ou podadas, que, após pesada, foi submetida à secagem, em estufa, a $65^{\circ} \mathrm{C}$, com ventilação forçada, até peso constante. Após secada, foi pesada novamente para determinação do teor de fitomassa seca e submetida às análises quanto à sua composição química, em termos de carbono orgânico, macro e micronutrientes.

Durante o cultivo não foi realizada adubação mineral ou orgânica, nem aplicação de defensivos agrícolas. As capinas foram realizadas com enxada, de acordo com o surgimento das plantas espontâneas. Em cada capina, uma amostra de plantas espontâneas foi submetida à secagem, em estufa, a $70{ }^{\circ} \mathrm{C}$, calculando-se, no final, a redução da infestação nos tratamentos. Quando necessário, foram realizadas irrigações semanais, por aspersão, de maneira a fornecer lâmina de água de cerca de $40 \mathrm{~mm}$ por semana. As irrigações foram suspensas aos 25 dias antes da colheita do taro.

A colheita do taro foi realizada aos nove meses após o plantio, com as plantas maduras, ou seja, com $95 \%$ das folhas senescidas. Na colheita, separaram-se os rizomasfilhos do rizoma-mãe; e eles foram classificados, com base no diâmetro, em filho grande, médio, pequeno e refugo, de acordo com Puiatti et al., (1992), contados e pesados para obtenção da massa de matéria fresca.

Após a colheita do taro, foram coletadas amostras de solo para análises químicas, em dois pontos de cada fileira central de taro, em cada repetição, na profundidade de 0-20 cm. Elas foram juntadas em amostra composta dos oito pontos de coleta de cada tratamento (quatro repetições $\mathrm{x}$ dois pontos de coleta em cada repetição).

Com base nos dados de produção de massa e dos teores de fitomassa seca e de nutrientes, nos respectivos cortes da crotalária (tratamentos), estimaram-se as quantidades de massa de matéria seca, de carbono orgânico e de macro e micronutrientes disponibilizados sobre o solo em cada tratamento. 
Tobela 1. Resultados das análises químicas de amostras de solos, realizadas à época do plantio e ao final da colheita do taro, nos tratamentos controle e épocas de corte das plantas de crotalária rente ao solo e podada na altura do dossel das plantas de taro, em dias após a semeadura da crotalária (DAS)

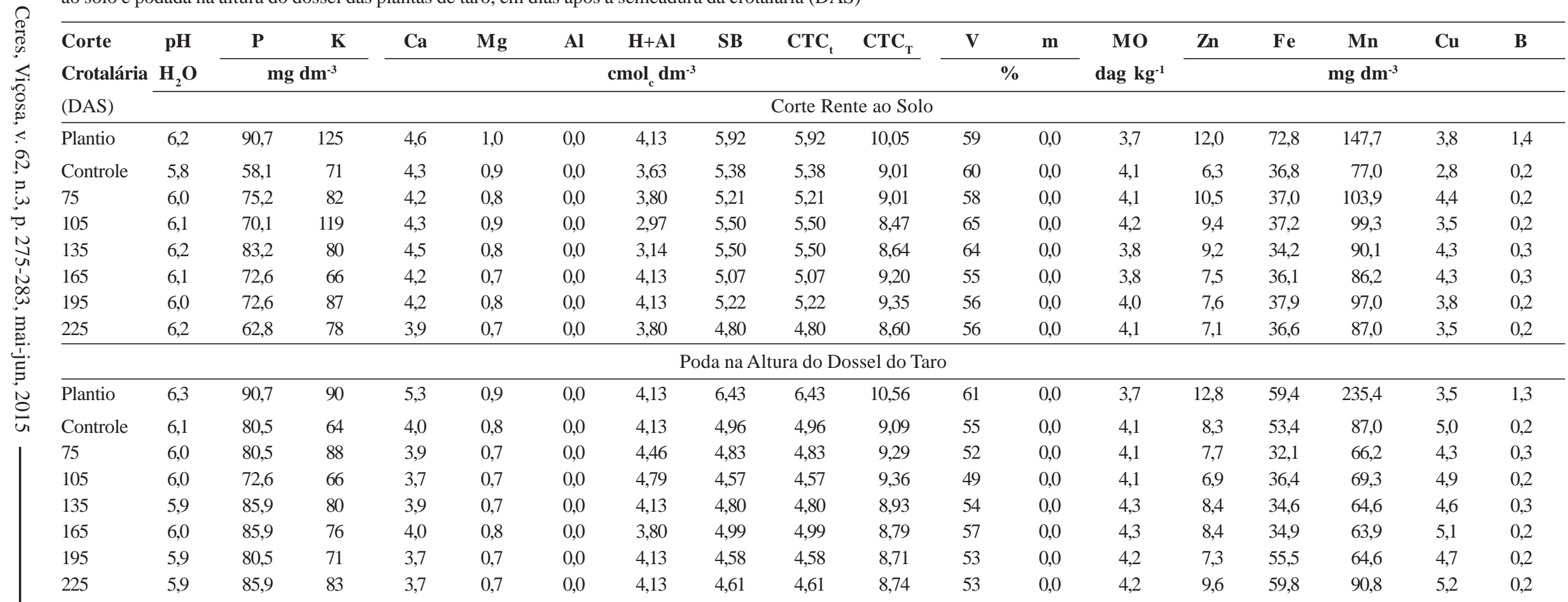

Cada valor é resultado da análise de amostra composta por oito pontos (4 repetições com 2 pontos amostrados em cada parcela). pH em água (1:2,5); $\mathrm{P}-\mathrm{K}-\mathrm{Fe}-\mathrm{Zn}-\mathrm{Mn}$ e $\mathrm{Cu}=\mathrm{Extrator} \mathrm{Mehlich} 1 ; \mathrm{Ca}-\mathrm{Mg}-\mathrm{Al}=\mathrm{Extrator}$ $\mathrm{KCl} 1 \mathrm{~mol} / \mathrm{L} ; \mathrm{H}+\mathrm{Al}=$ Extrator Acetato de $\mathrm{Ca} 0,5 \mathrm{~mol} / \mathrm{L} \mathrm{pH} 7,0 ; \mathrm{B}=$ água quente; $\mathrm{S}=$ Extrator - Fosfato monocálcico em ácido acético; $\mathrm{SB}=\mathrm{Soma}$ de bases; $\mathrm{CTC}(\mathrm{t})=\mathrm{Capacidade}$ de Troca Catiônica Efetiva; $\mathrm{CTC}(\mathrm{T})=$ Capacidade de Troca Catiônica a pH 7,0; V = Índice de Saturação por Bases; $m$ = Índice de Saturação por Alumínio; MO = matéria orgânica (C. orgânico x 1,724 - Walkley-Black). 
Os dados obtidos foram submetidos à análise de variância, com o programa computacional SAEG 9.1 (SAEG, 2007). Os dados em percentagem e em número foram transformados em arco-seno $\sqrt{x / 100}$ e $\sqrt{\mathrm{x}+1}$, respectivamente, para a realização da análise estatística. As médias referentes à produção de rizomas foram comparadas à do controle pelo teste de Dunnett e as médias referentes aos nutrientes foram comparadas, entre si, pelo teste de Tukey, ambos a 5\% de probabilidade.

\section{RESULTADOS E DISCUSSÃO}

Nas datas de realização dos cortes ou podas das plantas de crotalária, aos 75, 105, 135, 165, 195 e 225 DAS, as alturas das plantas de taro e de crotalária eram, em média, respectivamente, de: 1,03 e 2,$45 ; 1,55$ e 3,03 ; 1,54 e 3,$60 ; 1,40$ e 3,$60 ; 1,00$ e 3,$30 ; 0,15$ e 3,15 m. Por sua vez, no controle as plantas de taro apresentaram altura máxima de $1,15 \mathrm{~m}$, aos 150 dias após plantio. No taro, sob condições de limitação de luz incidente, há alongamento do pecíolo (Puiatti et al., 2000; Gondim et al., 2007; 2008), fato que explica a maior altura dessas plantas, quando em consórcio. A menor altura das plantas de taro, observada a partir dos 165 DAS, foi devida à senescência natural das folhas no processo de maturação.

Os tratamentos cujos cortes das plantas de crotalária (Experimento I) foram realizados aos 135, 165,195 e 225 DAS proporcionaram menor produção em massa de rizomas-mães (RM) e em número por planta de rizomas-filhos grandes (FG) e de filhos comerciais (FC), em comparação com o controle (Tabela 2). Resultados semelhantes foram observados quando apenas da poda da parte aérea das plantas de crotalária (Experimento II), apesar de a significância ter sido detectada para RM apenas para poda aos 195 DAS e, aos 135, 165 e 195 DAS, para número de FC. A menor produção em número de rizomas $\mathrm{FC}$ foi decorrente da menor produção em número de rizomas FG. Apesar disso, a produtividade, em $\mathrm{Mg} \mathrm{ha}^{-1}$ de rizomas FG e FC, não diferiu da do controle, indicando maior fitomassa média de rizoma FG. Portanto, o corte ou poda das plantas de crotalária, após os 105 DAS, não seria interessante para o agricultor, se for analisada apenas a produção, em massa, de rizomas-mães e em número de rizomas FG e FC. Por sua vez, o corte aos 75 DAS (Experimento I) e, principalmente, o tratamento de poda aos 75 e 105 DAS (Experimento II) foram vantajosos, em termos de produção de rizomas maiores (FG), refletindo-se na produção de rizomas FC, apesar de não diferirem do controle (Tabela 2).

Os cortes das plantas de crotalária, realizados aos 135 e 165 , e as podas, realizadas aos 135,165 e aos 195
DAS, foram os que, de modo geral, propiciaram maiores quantidades de fitomassas fresca e seca de crotalária, de $\mathrm{C}$ e de macro e micronutrientes (Tabela 3); esse aspecto é relevante, em se pensando na manutenção da fertilidade do solo para cultivos posteriores.

A relação $\mathrm{C}: \mathrm{N}$ da crotalária cortada ou podada, que esteve, em média, em torno de 25:1 e de 22:1 nos quatro primeiro cortes ou podas, respectivamente, alcançou valores, respectivamente, de 41:1 e 33:1 nas duas últimas podas. Esse aumento da relação $\mathrm{C}: \mathrm{N}$ nas duas últimas avaliações foi decorrente do processo de maturação das plantas de crotalária, com queda das folhas, restando, praticamente, somente o caule principal e os ramos laterais, estruturas altamente lignificadas. Portanto, é possível que, em razão da grande massa de crotalária depositada sobre o solo, especialmente nas podas aos135 e 165 DAS, e da elevada relação C:N nas duas últimas podas, tenha ocorrido imobilização de $\mathrm{N}$ durante o processo de mineralização da biomassa de crotalária disponibilizada sobre o solo, o que pode ter afetado o crescimento das plantas de taro, refletindose na menor produção de RM, FG e FC, comparado ao controle.

Além do possível processo de imobilização do $\mathrm{N}$ do solo, outro fator que pode ter contribuído para a menor produção de rizomas de taro no consórcio, sobretudo quando as plantas de crotalária foram cortadas ou podadas após os 105 DAS, pode ter sido o sombreamento excessivo das plantas de taro, promovido pela crotalária. As plantas de crotalária apresentaram crescimento exuberante, alcançando, já no corte ou poda, aos 75 DAS, mais do dobro da altura das plantas de taro. Gondim et al. (2007) verificaram que o aumento da intensidade de sombra artificial, de 0 para $50 \%$, causou redução da produtividade do taro e que intensidade de sombra de até $18 \%$, durante o ciclo todo, ou nos três primeiros meses, ou do $3^{\circ}$ ao $6^{\circ}$ mês após o plantio, pouco afetou o desenvolvimento das plantas de taro e a produção de rizomas

Plantas de taro sob condições de sombreamento privilegiam o investimento na formação da parte aérea, em detrimento da subterrânea, na busca pela captação de luz (Oliveira et al., 2006; Gondim et al., 2008). De acordo com Gondim et al. (2008), uma das possíveis causas da menor produtividade da cultura do taro, quando sombreada, é redução da espessura da folha e da densidade de estômatos, que o sombreamento provoca no taro. Portanto, com base nessas informações e nos resultados obtidos com a produção de taro, neste trabalho, o corte ou poda das plantas de crotalária deve ser iniciado mais precocemente e, ou a implantação da associação poderia ser realizada com as plantas de taro mais desenvolvidas. 
حृ Tabela 2. Produtividade $\left(\mathrm{t} \mathrm{ha}^{-1}\right)$ e número por planta (No/pl.) de rizomas-mães (RM) e de filhos grande (FG), médio (FM), pequeno (FP), refugo (FR), comercial (FC) e total (PT) de taro 'Japonês', nos tratamentos controle e épocas de corte das plantas de crotalária rente ao solo e podada na altura do dossel das plantas de taro, em dias após a semeadura da crotalária (DAS)

\begin{tabular}{|c|c|c|c|c|c|c|c|c|c|c|c|c|c|}
\hline \multirow{2}{*}{$\begin{array}{l}\text { Corte } \\
\text { (DAS) }\end{array}$} & \multirow{2}{*}{$\begin{array}{c}\text { RM } \\
\text { t ha }^{-1} \\
\end{array}$} & \multicolumn{2}{|c|}{ FG } & \multicolumn{2}{|c|}{ FM } & \multicolumn{2}{|c|}{$\mathbf{F P}$} & \multicolumn{2}{|c|}{ FR } & \multicolumn{2}{|c|}{ FC } & \multicolumn{2}{|c|}{ PT } \\
\hline & & t ha $^{-1}$ & N\%/pl. & t ha ${ }^{-1}$ & N\%/pl. & t ha $^{-1}$ & No/pl. & t ha $^{-1}$ & N\%/pl. & t ha $^{-1}$ & N\%/pl. & t ha $^{-1}$ & $\mathrm{~N} / \mathrm{pl}$. \\
\hline & \multicolumn{13}{|c|}{ Corte Rente ao Solo } \\
\hline Controle & 12,43 & 5,01 & 0,72 & 3,48 & 2,38 & 2,11 & 3,46 & 0,90 & 3,13 & 10,06 & 6,57 & 23,39 & 9,69 \\
\hline 75 & 11,67 & 5,55 & 0,50 & 3,34 & 2,42 & 1,91 & 2,84 & 0,89 & 2,50 & 10,80 & 5,75 & 22,48 & 8,25 \\
\hline 105 & 12,08 & 3,23 & 0,50 & 3,81 & 1,79 & 2,05 & 2,42 & 0,71 & 3,63 & 9,09 & 4,71 & 21,17 & 8,33 \\
\hline 135 & $8,51^{*}$ & 3,33 & $0,04 *$ & 3,64 & 1,58 & 2,15 & 2,83 & 0,77 & 3,29 & 9,12 & $4,45^{*}$ & 17,63 & 7,74 \\
\hline 165 & $9,70^{*}$ & 4,79 & $0,12^{*}$ & 3,52 & 0,79 & 1,77 & 2,87 & 0,85 & 3,28 & 10,07 & $3,78^{*}$ & 19,77 & 7,07 \\
\hline 195 & $8,50 *$ & 3,00 & $0,17^{*}$ & 4,44 & 1,66 & 2,07 & 2,33 & 0,83 & 3,16 & 9,52 & $4,16^{*}$ & 18,02 & 7,32 \\
\hline 225 & $9,12 *$ & 4,97 & $0,24 *$ & 3,30 & 1,41 & 1,85 & 2,12 & 0,86 & 3,04 & 10,13 & $3,77^{*}$ & 19,25 & 6,81 \\
\hline \multirow[t]{2}{*}{$\mathrm{CV}(\%)$} & 25,7 & 70,8 & 86,8 & 24,5 & 54,3 & 14,2 & 21,7 & 13,0 & 26,1 & 29,4 & 27,7 & 22,7 & 21,2 \\
\hline & \multicolumn{13}{|c|}{ Poda na Altura do Dossel do Taro } \\
\hline Controle & 9,73 & 4,35 & 0,42 & 3,37 & 1,54 & 1,96 & 3,92 & 0,92 & 3,62 & 9,68 & 5,87 & 20,37 & 9,49 \\
\hline 75 & 10,62 & 6,63 & 0,25 & 3,83 & 1,33 & 2,07 & 3,00 & 0,90 & 2,75 & 12,53 & 4,57 & 24,05 & 7,32 \\
\hline 105 & 11,48 & 8,27 & 0,41 & 3,58 & 1,62 & 2,01 & 3,04 & 0,85 & 2,50 & 13,86 & 5,07 & 26,19 & 7,57 \\
\hline 135 & 8,81 & 2,78 & 0,25 & 3,56 & 0,83 & 2,00 & 2,46 & 0,76 & 3,12 & 8,34 & $3,53^{*}$ & 17,91 & 6,65 \\
\hline 165 & 7,78 & 4,27 & 0,13 & 2,69 & 0,91 & 1,90 & 3,04 & 0,96 & 2,79 & 8,86 & $4,08^{*}$ & 17,60 & 6,87 \\
\hline 195 & $7,17 *$ & 3,92 & 0,10 & 3,29 & 0,96 & 1,81 & 2,50 & 0,81 & 4,12 & 9,02 & $3,53 *$ & 17,00 & 7,66 \\
\hline 225 & 8,02 & 1,56 & 0,17 & 3,52 & 1,17 & 1,94 & 3,24 & 0,83 & 3,33 & 7,02 & 4,58 & 15,86 & 7,91 \\
\hline $\mathrm{CV}(\%)$ & 17,8 & 81,1 & 100,0 & 23,5 & 37,2 & 11,3 & 25,1 & 12,3 & 33,2 & 39,5 & 18,8 & 25,1 & 17,8 \\
\hline
\end{tabular}

Médias, nas colunas, seguidas de asterisco (*), diferem do controle pelo teste de Dunnett a 5\% de probabilidade. 
Tabela 3. Teores de carbono (C) e de matéria seca (MS) e quantidade de massas de matéria fresca (MF), seca (MS), C e de macro (N, P, K, Ca, Mg e S) e micronutrientes (Zn, Fe, Mn, Cu e B), depositadas sobre o solo pelas plantas de crotalária cortadas rente ao solo e podada na altura do dossel das plantas de taro, em dias após a semeadura da crotalária (DAS)

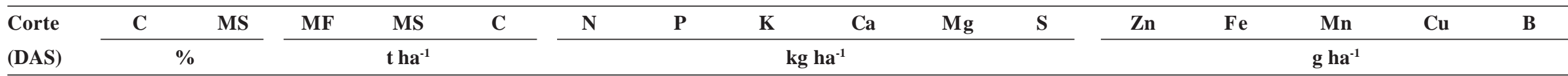

Corte Rente ao Solo

\begin{tabular}{|c|c|c|c|c|c|c|c|c|c|c|c|c|c|c|c|c|}
\hline Controle & - & - & - & - & - & - & - & - & - & - & - & - & - & - & - & - \\
\hline 75 & 52,66 & 25,6 & $16,95 \mathrm{c}$ & $4,34 \mathrm{~d}$ & $2,28 \mathrm{~d}$ & $87,19 \mathrm{c}$ & $9,38 \mathrm{c}$ & $69,88 \mathrm{c}$ & $29,95 \mathrm{c}$ & $11,54 \mathrm{c}$ & $3,39 \mathrm{~d}$ & $69,01 \mathrm{~d}$ & $311,62 d$ & $170,57 \mathrm{~d}$ & $27,34 \mathrm{c}$ & $124,56 b$ \\
\hline 105 & 52,26 & 25,8 & $32,25 \mathrm{ab}$ & $8,32 \mathrm{c}$ & $4,35 \mathrm{c}$ & $163,32 \mathrm{~b}$ & $20,65 b$ & $176,80 \mathrm{~b}$ & $61,82 \mathrm{~b}$ & $22,46 b$ & $6,74 \mathrm{c}$ & $222,98 \mathrm{bc}$ & $836,16 \mathrm{~cd}$ & $443,46 \mathrm{c}$ & $49,09 \mathrm{bc}$ & $206,34 b$ \\
\hline 135 & 52,69 & 35,4 & $36,05 \mathrm{a}$ & $12,78 \mathrm{ab}$ & $6,73 \mathrm{ab}$ & $280,52 \mathrm{a}$ & $27,23 \mathrm{ab}$ & $244,48 \mathrm{a}$ & $85,25 \mathrm{a}$ & $27,22 b$ & $10,22 \mathrm{~b}$ & $270,94 \mathrm{ab}$ & $3.736,87 \mathrm{a}$ & $503,53 \mathrm{bc}$ & 86,94 a & $457,52 \mathrm{a}$ \\
\hline 165 & 52,61 & 43,3 & $33,18 \mathrm{ab}$ & $14,38 \mathrm{a}$ & $7,56 \mathrm{a}$ & $308,88 \mathrm{a}$ & $33,72 \mathrm{a}$ & $262,00 \mathrm{a}$ & $86,28 \mathrm{a}$ & $36,67 \mathrm{a}$ & $16,97 \mathrm{a}$ & $353,75 \mathrm{a}$ & $1.531,47 \mathrm{~b}$ & $1.006,60 \mathrm{a}$ & $86,28 \mathrm{a}$ & $478,85 \mathrm{a}$ \\
\hline 195 & 53,82 & 43,6 & $24,53 \mathrm{bc}$ & $10,68 b c$ & $5,74 \mathrm{abc}$ & $132,00 \mathrm{bc}$ & $13,16 \mathrm{c}$ & $94,30 \mathrm{c}$ & $35,46 \mathrm{c}$ & $19,97 \mathrm{~b}$ & $8,12 \mathrm{bc}$ & $162,26 \mathrm{c}$ & $1.211,11 \mathrm{bc}$ & $501,96 \mathrm{bc}$ & $57,67 \mathrm{~b}$ & $185,83 \mathrm{~b}$ \\
\hline 225 & 52,68 & 48,3 & $19,70 \mathrm{c}$ & $9,53 b c$ & $5,02 \mathrm{bc}$ & $128,18 \mathrm{bc}$ & $9,66 \mathrm{c}$ & $127,42 \mathrm{bc}$ & $45,94 \mathrm{bc}$ & $19,73 \mathrm{~b}$ & $7,53 \mathrm{bc}$ & $297,34 \mathrm{ab}$ & $1.203,64 \mathrm{bc}$ & $641,37 \mathrm{~b}$ & $86,72 \mathrm{a}$ & $372,62 \mathrm{a}$ \\
\hline $\mathrm{CV}(\%)$ & - & - & 14,8 & 15,1 & 15,1 & 15,4 & 15,3 & 15,6 & 15,5 & 14,8 & 15,1 & 16,1 & 18,0 & 15,6 & 16,2 & 16,5 \\
\hline
\end{tabular}

Poda na Altura do Dossel do Taro

\begin{tabular}{|c|c|c|c|c|c|c|c|c|c|c|c|c|c|c|c|c|}
\hline Controle & - & - & - & - & - & - & - & - & - & - & - & - & - & - & - & - \\
\hline 75 & 51,21 & 22,2 & $7,58 \mathrm{c}$ & $1,68 \mathrm{c}$ & $0,86 \mathrm{~d}$ & $52,45 \mathrm{c}$ & $1,65 \mathrm{c}$ & $31,12 \mathrm{c}$ & $19,37 \mathrm{c}$ & $6,65 \mathrm{~d}$ & $2,27 \mathrm{~d}$ & $43,85 \mathrm{~d}$ & $131,54 \mathrm{~d}$ & $99,12 \mathrm{e}$ & $15,62 \mathrm{c}$ & $149,90 \mathrm{~cd}$ \\
\hline 105 & 50,96 & 23,9 & $15,88 \mathrm{ab}$ & $3,79 \mathrm{bc}$ & $1,93 \mathrm{c}$ & $146,45 b$ & $3,35 \mathrm{~b}$ & $78,23 \mathrm{~b}$ & $41,69 b$ & $13,49 \mathrm{c}$ & $5,04 \mathrm{c}$ & $152,74 \mathrm{c}$ & $698,88 \mathrm{c}$ & $289,94 \mathrm{~d}$ & $71,42 \mathrm{a}$ & $174,72 \mathrm{c}$ \\
\hline 135 & 52,40 & 45,4 & $21,00 \mathrm{a}$ & $9,20 \mathrm{a}$ & $4,82 \mathrm{a}$ & 201,94 a & $7,61 \mathrm{a}$ & $164,86 \mathrm{a}$ & 68,17 a & $20,79 b$ & $7,54 \mathrm{~b}$ & $205,16 b$ & $998,20 \mathrm{~b}$ & $552,92 \mathrm{~b}$ & $53,36 \mathrm{~b}$ & $291,64 \mathrm{ab}$ \\
\hline 165 & 52,65 & 56,2 & $15,25 \mathrm{~b}$ & $8,70 \mathrm{a}$ & $4,58 \mathrm{a}$ & $157,30 \mathrm{~b}$ & $7,83 \mathrm{a}$ & $97,88 \mathrm{~b}$ & $63,60 \mathrm{a}$ & $27,23 \mathrm{a}$ & $9,05 \mathrm{a}$ & $175,74 \mathrm{c}$ & $1.077,93 \mathrm{ab}$ & $675,12 \mathrm{a}$ & $61,77 \mathrm{ab}$ & $306,24 \mathrm{a}$ \\
\hline 195 & 52,58 & 57,8 & $15,03 \mathrm{~b}$ & $8,73 \mathrm{a}$ & $4,59 \mathrm{a}$ & $195,64 \mathrm{~b}$ & $8,15 \mathrm{a}$ & $108,78 \mathrm{ab}$ & $60,76 \mathrm{a}$ & $22,44 a b$ & $8,38 \mathrm{ab}$ & $317,77 \mathrm{a}$ & $1.388,94 \mathrm{a}$ & $461,82 \mathrm{c}$ & $68,97 \mathrm{a}$ & $250,55 \mathrm{~b}$ \\
\hline 225 & 54,39 & 97,1 & $7,23 \mathrm{c}$ & $7,00 \mathrm{ab}$ & $3,81 \mathrm{ab}$ & $56,30 \mathrm{c}$ & $4,43 \mathrm{~b}$ & 54,25 & $15,96 \mathrm{c}$ & $7,21 \mathrm{~d}$ & $4,06 \mathrm{c}$ & $90,30 \mathrm{~d}$ & $940,10 \mathrm{~b}$ & $347,20 \mathrm{~d}$ & $26,60 \mathrm{c}$ & $105,00 \mathrm{~d}$ \\
\hline CV (\%) & - & - & 18,1 & 23,7 & 14,2 & 17,6 & 13,8 & 18,3 & 13,3 & 14,2 & 15,6 & 13,7 & 15,0 & 14,4 & 16,0 & 15,4 \\
\hline
\end{tabular}

Médias, nas colunas, seguidas da mesma letra, não diferem entre si pelo teste de Tukey a 5\% de probabilidade.

$\mathrm{P}-\mathrm{K}-\mathrm{Fe}-\mathrm{Zn}-\mathrm{Mn} \mathrm{e} \mathrm{Cu}=$ Extrator Mehlich 1; $\mathrm{Ca}-\mathrm{Mg}=$ Extrator $\mathrm{KCl} 1 \mathrm{~mol} / \mathrm{L} ; \mathrm{B}=$ água quente; $\mathrm{S}=$ Extrator - Fosfato monocálcico em ácido acético; $\mathrm{C}=$ Carbono orgânico - Walkley-Black; $\mathrm{N}=$ digestão sulfúrica - método Kjeldah 
Neste trabalho, o aporte verificado de biomassa seca e de $\mathrm{N}$ e a ciclagem de nutrientes, na média, foram superiores às $6,58 \mathrm{t} \mathrm{ha}^{-1}$ de biomassa seca, $159 \mathrm{~kg} \mathrm{ha}^{-1} \mathrm{de} \mathrm{N}$, $20 \mathrm{~kg} \mathrm{ha}^{-1}$ de P, $136 \mathrm{~kg} \mathrm{ha}^{-1}$ de K, $64 \mathrm{~kg} \mathrm{ha}^{-1}$ de Ca e de 16 $\mathrm{kg} \mathrm{ha}^{-1}$ de $\mathrm{Mg}$ obtidos com plantas de guandu (Cajanus cajan), cultivado, em faixas, com taro (Oliveira et al., 2006). Esses valores indicam o grande potencial de uso da crotalária em associação com a cultura do taro, como adubação verde, principalmente visando a cultivos subsequentes. Portanto, mesmo que no consórcio não haja benefícios à produção da cultura consorciada (taro, no presente caso), o efeito residual dos adubos verdes, visando à manutenção da fertilidade para cultivos posteriores é de fundamental importância, como apontado nos trabalhos de Vargas (2009) Diniz (2011) e Souza \& Guimarães (2013).

Apesar de não aumentar a produção de rizomas, a associação do taro com faixas de guandu (Oliveira et al., 2006) ou em consórcio com crotalária (Oliveira et al., 2004) apresenta a vantagem adicional de proteção das plantas de taro contra a queimadura foliar pela radiação solar, no verão. Além disso, conforme observado por esses autores, o material disposto sobre o solo foi eficaz no controle de plantas espontâneas, reduzindo o gasto com mão de obra para capinas.

Neste trabalho, não foi observada queimadura foliar nas plantas de taro; todavia, a associação com crotalária reduziu a infestação por plantas espontâneas. Em média, comparando-se com o controle, os tratamentos de consórcio reduziram em $44 \%$ a massa de matéria seca de plantas espontâneas. Esse efeito é importante, pois, a mão de obra com capinas na cultura do taro pode representar até $25 \%$ do custo de produção (Heredia Zarate et al., 2012).

Ao analisar a composição química de amostras de solo retiradas no final do ciclo da cultura do taro, observa-se redução dos valores de $\mathrm{K}$ e $\mathrm{Ca}$, enquanto as demais características mantiveram-se como estavam ou tiveram pequenas oscilações, em comparação com o momento do plantio (Tabela 1). A cultura do taro promove grande extração de nutrientes, sobretudo de $\mathrm{K}, \mathrm{N}$ e $\mathrm{Ca}$ (Puiatti et al., 1992). De acordo com Costa et al. (2014), o K é rapidamente disponibilizado na fitomassa fragmentada. Em trabalho de consórcio entre crotalária e milheto, na fitomassa dessecada e fragmentada aos 75 DAS, esses autores observaram, aos 91 dias após a fragmentação, que restou apenas 1,5\% da quantidade de K presente na fitomassa inicial. Portanto, é possível que os minerais, liberados durante a mineralização da crotalária depositada sobre o solo nas primeiras épocas de corte ou poda, tenham sido absorvidos pelas plantas de taro, embora, neste trabalho, a planta cortada e as partes podadas não não tenham sido fragmentadas, como no trabalho de Costa et al. (2014).
Após a colheita do taro, ainda havia sobre o solo grande quantidade de biomassa de crotalária a ser mineralizada, o que também pode explicar a ausência de elevação dos valores de minerais nas amostras de solo coletadas. Todavia, em média, observou-se aumento de $8,4 \%$ do teor de MO, em comparação com o da época do plantio (Tabela 1). Embora parte dessa massa seja composta por restos da cultura do taro, se for considerado que a maior parcela do $\mathrm{C}$ fixado na $\mathrm{MO}$ veio da crotalária, esta fixou de 2,28 a 7,56 $\mathrm{Mg} \mathrm{ha}^{-1}$, quando cortada, e de 0,86 a 4,82 $\mathrm{Mg} \mathrm{ha}^{-1}$, quando podada (Tabela 3). Esse carbono pode ser muito significativo para a microbiota e a fertilidade do solo, em cultivos posteriores.

$\mathrm{O}$ aporte de $\mathrm{N}$ ao solo pela crotalária variou, de acordo com o tratamento, de 87,19 a $308,88 \mathrm{~kg} \mathrm{ha}^{-1}$, quando cortada, e de 52,45 a 201,94 $\mathrm{kg} \mathrm{ha}^{-1}$, quando podada (Tabela 3 ). Se for considerado que $57 \%$ desse $\mathrm{N}$ foram devidos à FBN (Perin et al., 2004), a crotalária permitiu incremento de $\mathrm{N}$ ao solo da ordem de 46,7 a 176,1 $\mathrm{kg} \mathrm{ha}^{-1}$ (quando cortada) e de 30,90 a 115,1 kg ha-1 (quando podada), valores esses muito significativos, pois representam economia com gasto de fertilizantes nitrogenados. Além disso, a ciclagem dos minerais e a melhoria dos aspectos físicos do solo, beneficiando cultivos subsequentes, não devem ser menosprezadas, conforme efeito residual da adubação verde verificado nas culturas de repolho (Vargas, 2009), brócolis, abobrinha e milho (Diniz, 2011).

Pelos resultados obtidos neste trabalho, é agronomicamente viável a associação da crotalária com a cultura do taro. Todavia, considerando-se apenas a produção de rizomas de taro, não seria indicado o corte ou a poda das plantas de Crotalaria juncea após os 105 dias de sua semeadura, ou 160 dias após o plantio do taro.

\section{CONCLUSÕES}

A consorciação do taro com crotalária é viável, sendo que o corte ou a sua poda deve ser realizado, preferencialmente, até aos 105 dias após a semeadura (160 dias após o plantio do taro), para maior produtividade de rizomas grandes e comerciáveis.

Maiores quantidades de fitomassas fresca e seca, de carbono e de nutrientes são proporcionados pela crotalária nos cortes ou podas realizados aos 135 e 165 dias após a semeadura, com aportes de $\mathrm{N}$ de $308 \mathrm{~kg} \mathrm{ha}^{-1}$, no corte aos 165 , e de $202 \mathrm{~kg} \mathrm{ha}^{-1}$, na poda realizada aos 135 dias após a semeadura.

\section{AGRADECIMENTOS}

Os autores agradecem ao CNPQ (Conselho Nacional de Desenvolvimento Científico e Tecnológico) e à FAPEMIG (Fundação de Amparo à Pesquisa do Estado de Minas Gerais), pelo apoio ao projeto e à publicação. 


\section{REFERÊNCIAS}

Costa CHM, Crusciol CAC, Soratto RP \& Neto JF (2014) Persistência e liberação de elementos da fitomassa do consórcio crotalária com milheto sob fragmentação. Revista Ciência Agronômica, 45:197-208.

Diniz ER (2011) Efeitos de doses de adubo verde em cultivos sucessivos de brócolis, abobrinha e milho. Tese de doutorado. Universidade Federal de Viçosa, Viçosa. 92p.

Embrapa (2010) A agricultura familiar no contexto mundial. Disponível em: <http://www.embrapa.br/embrapa/imprensa/artigos/2006/artigo.2006-10-24.2094779241>. Acessado em: 18 de Fevereiro de 2010.

FAO (2013) FAO statistical database. Disponível em: < htpp// www.fao.org/>. Acessado em: 03 de dezembro 2013.

Gondim ARO, Puiatti M, Cecon PR \& Finger F (2007) Crescimento, partição de fotoassimilados e produção de rizomas em taro cultivado sob sombreamento artificial. Horticultura Brasileira, 25:418-428.

Gondim ARO, Puiatti M, Ventrella MC \& Cecon PR (2008) Plasticidade anatômica da folha de taro cultivado sob diferentes condições de sombreamento. Bragantia, 67:1037-1045.

Heredia Zárate NA, Vieira MC, Oliveira ACP \& Lima AA (2005) Produção e renda bruta de dois cultivares de taro, em cultivo solteiro e consorciado com alface. Semina: Ciências Agrárias, 26:283-290.

Heredia Zárate NA, Vieira MC, Oliveira ACP \& Lima AA (2012) Produção agroeconômica de taro em função do número de amontoas. Semina: Ciências Agrárias, 33:1673-1680.

Oliveira FL, Guerra JGM, Junqueira RM, Silva EE, Oliveira FF, Espindola JAA, Almeida DL, Ribeiro RLD \& Urquiaga S (2006) Crescimento e produtividade do inhame cultivado entre faixas de guandu em sistema orgânico. Horticultura Brasileira, 24:53-58.

Oliveira FL, Ribeiro RLD, Silva VV, Guerra JGM \& Almeida DL (2004) Desempenho do inhame (taro) em plantio direto e no consórcio com crotalária, sob manejo orgânico. Horticultura Brasileira, 22:638-641.

Perin A, Santos RHS, Urquiaga S, Guerra JGM \& Cecon PR (2004) Produção de fitomassa, acúmulo de nutrientes e fixação biológica de nitrogênio por adubos verdes em cultivo isolado e consorciado. Pesquisa Agropecuária Brasileira, 39:35-40.

Puiatti M, Fávero C, Finger FL \& Gomes JM (2000) Crescimento e produtividade de inhame e de milho doce em cultivo associado. Horticultura Brasileira, 18:24-30.

Puiatti M, Greeman S, Katsumoto R \& Fávero C (1992) Crescimento e absorção de macronutrientes pelo inhame 'Chinês' e 'Japonês'. Horticultura Brasileira, 10:89-92.

Puiatti M \& Pereira FHF (2007) Taro [Colocasia esculenta (L.) Schott]. In: Paula Júnior TJ \& Venzon M (Eds.) 101 Culturas: Manual de Tecnologias Agrícolas. $1^{a}$ ed. Belo Horizonte, Epamig. p.729-734.

SAEG (2007) Sistema para Análises Estatísticas. Versão 9.1. Viçosa, Fundação Arthur Bernardes - UFV. CD-ROM.

Souza JL \& Guimarães GP (2013) Rendimento de massa de adubos verdes e o impacto na fertilidade do solo em sucessão de cultivos orgânicos. Bioscience Journal, 29:1796-1805.

Vandermeer JH (1989) The ecology of intercropping. Cambridge, Cambridge University Press. 237p.

Vargas TO (2009) Contribuição da raiz e da parte aérea de duas leguminosas de adubação verde na produção do repolho. Dissertação de Mestrado. Universidade Federal de Viçosa, Viçosa. 75p. 